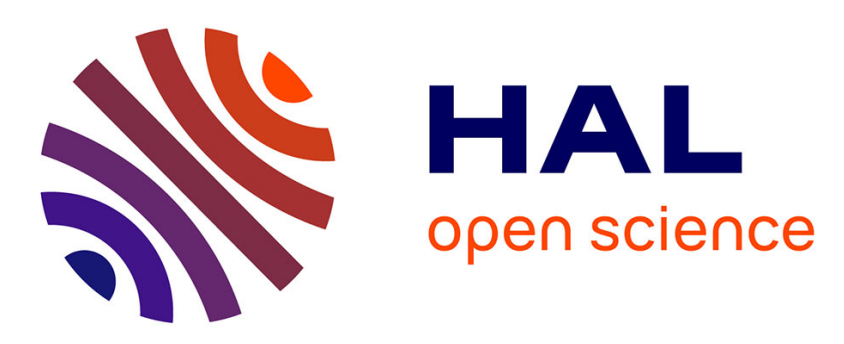

\title{
Disturbance attenuation and trajectory tracking via a reduced-order output feedback controller for robot manipulators
}

Michel Zasadzinski, Edouard Richard, Mohamed Fayçal Khelfi, Mohamed Darouach

\section{To cite this version:}

Michel Zasadzinski, Edouard Richard, Mohamed Fayçal Khelfi, Mohamed Darouach. Disturbance attenuation and trajectory tracking via a reduced-order output feedback controller for robot manipulators. Automatica, 1998, 34 (12), pp.1539-1546. 10.1016/S0005-1098(03)00006-2 . hal-00098139

\section{HAL Id: hal-00098139 \\ https://hal.science/hal-00098139}

Submitted on 24 Sep 2006

HAL is a multi-disciplinary open access archive for the deposit and dissemination of scientific research documents, whether they are published or not. The documents may come from teaching and research institutions in France or abroad, or from public or private research centers.
L'archive ouverte pluridisciplinaire HAL, est destinée au dépôt et à la diffusion de documents scientifiques de niveau recherche, publiés ou non, émanant des établissements d'enseignement et de recherche français ou étrangers, des laboratoires publics ou privés. 


\title{
Disturbance attenuation and trajectory tracking via a reduced-order output feedback controller for robot manipulators
}

\author{
M. Zasadzinski ${ }^{\dagger}$, E. Richard ${ }^{\dagger \dagger}$, M.F. Khelfi ${ }^{\dagger}$ and M. Darouach ${ }^{\dagger}$ \\ ${ }^{\dagger} C R A N-C N R S$ UPRES-A 7039 \\ I.U.T de Longwy - Université Henri Poincaré - Nancy I \\ 186, rue de Lorraine, 54400 Cosnes et Romain, FRANCE \\ Tel : (0033) 3 82 259113 - Fax: (0033) 382259117 \\ E-mail : mzasad@iut-longwy.u-nancy.fr \\ ‡ INRIA Lorraine (CONGE Project), 4 rue Marconi, 57070 Metz, FRANCE \\ \# Institut d'Informatique - Université d'Oran, Es Senia, ALGERIE
}

\begin{abstract}
This paper presents a solution to the problem of trajectory tracking with external disturbance attenuation in robotics systems via a reduced-order output feedback controller, without velocity measurement. The proposed control law ensures both asymptotic stability of the closed-loop system and external disturbance attenuation. The approach is based on the notion of the $L_{2}$-gain and requires to solve two algebraic Riccati inequalities. The proposed controller design is illustrated by a simulation example.
\end{abstract}

Keywords. Rigid robot manipulator, Reduced-order output feedback controller, Trajectory tracking, Asymptotic stability, $L_{2}$-gain, Disturbance attenuation, Algebraic Riccati inequalities.

\section{Introduction}

The design of robot controllers [LEW 93, SPO 89] is usually based on complete state feedback, the measurements of both joint angular positions and joint angular velocities are required. The joint angular position measurements can be obtained by means of encoders or resolvers, which can give very accurate measurements of the joint displacements. But, joint angular velocities obtained from of tachometers are often contaminated by noise. One solution to this problem is to reconstruct the joint angular velocity signal via an observer [BER 93a, BER 93b, CAN 92, KHE 96, NIC 93] and then to use the estimated signal in the control loop.

The problem of external disturbance attenuation was not discussed in these references. For linear systems, the $H_{\infty}$ control theory has been revealed to be a powerful tool to solve the disturbance attenuation problem. By solving two coupled algebraic Riccati equalities, full-order $H_{\infty}$ output feedback compensators were given first in [DOY 89] and [SAM 90]. In [HSU 94], a reduced-order version was proposed. For the nonlinear case, an approach based on Hamilton-Jacobi equalities was introduced in [VDS 92], [ISI 92] and [KRE 94] to develop an $H_{\infty}$ state feedback controller and an $H_{\infty}$ output feedback controller, respectively. In the nonlinear case, the notion of $L_{2}$-gain generalizes the $H_{\infty}$ norm of a linear transfer function. Note that, in these works, the controller is obtained by solving equations (of Riccati (linear case) or Hamilton-Jacobi (nonlinear case) types) while the disturbance attenuation constraint requires only to solve inequalities. The use of the $L_{2}$-gain approach in the nonlinear disturbance attenuation problem is treated in Knobloch et al. [KNO 93], van der Schaft [VDS 96] and references therein.

Recently, in [AST 94] the nonlinear $H_{\infty}$ control theory was used in robotics control to treat the problem of state feedback stabilization in the presence of unknown torque disturbances and to derive an upper 
bound on the $L_{2}$-gain of the closed-loop system. Point-to-point control for a robot manipulator with disturbance attenuation has been treated in [ZAS 96] via a reduced-order output feedback controller.

In this paper, we present a systematic method to design a reduced-order output feedback controller for rigid robot manipulators. The proposed controller provides a solution to the problem of external disturbance attenuation in a $L_{2}$-gain sense and guarantees an asymptotic stability of the closed-loop system with a trajectory tracking control for an n-degrees-of-freedom ( $n$-D.O.F) rigid robot manipulator. By using the Lipschitz property of the robot model, the controller design is based on the solution of two algebraic Riccati inequalities. This approach avoids to need to solve a Hamilton-Jacobi equation. Note that the solution of a Hamilton-Jacobi equation is often obtained by means of a linearization technique for which the neighborhood of validity is unknown [VDS 92].

This paper is organized as follows. In section 2, we recall the dynamic model of an $n$-D.O.F rigid robot manipulator, define the reduced-order controller structure dedicated to the trajectory tracking objective, and formulate the problem of external disturbance attenuation (in a $L_{2}$-gain sense). In section 3 , we show that the external disturbance attenuation can be ensured by an algebraic Riccati inequality, and that this inequality implies the closed-loop asymptotic stability. Then a solution of this algebraic Riccati inequality is given. For this control law, a domain contained into the attraction domain is determined. The proposed control design procedure is summarized in section 4 and illustrated by a simulation example in section 5 .

Notation. Throughout this paper, $\|x(t)\|=\sqrt{x^{T}(t) x(t)}$ denotes the Euclidean vector norm and $L_{2}[0, \infty)$ represents the space of square integrable functions over $[0, \infty): x(t) \in L_{2}[0, \infty)$ if $\int_{0}^{\infty}\|x(t)\|^{2} d t<\infty$. $I_{n}$ denotes the $(n \times n)$ identity matrix, the subscript is dropped if no confusion may arise.

\section{Problem Formulation}

The equations of motion of an n-link rigid robot manipulator in the joint space and in the presence of external disturbances can be written as [SPO 89]

$$
H(q) \ddot{q}+C(q, \dot{q}) \dot{q}+F_{v} \dot{q}+G(q)=\Gamma+w_{0}
$$

where $q \in \mathbb{R}^{n}$ is the vector of generalized joint coordinates, $\Gamma \in \mathbb{R}^{n}$ is the vector of torques/forces acting at each joint, $w_{0} \in \mathbb{R}^{n}$ denotes the vector of external disturbances, $H(q) \in \mathbb{R}^{n \times n}$ is the symmetric and positive definite inertia matrix, $C(q, \dot{q}) \dot{q} \in \mathbb{R}^{n}$ represents the centrifugal and Coriolis forces, $F_{v} \in \mathbb{R}^{n \times n}$ is the diagonal matrix of the viscous coefficients, $G(q) \in \mathbb{R}^{n}$ is the vector of gravitational forces. The dynamic equation (1) has the following properties [LEW 93] which are used in the control design

$$
\begin{aligned}
& h_{1} I \leqslant H(q) \leqslant h_{2} I \text { with } h_{1}>0 \text { and } h_{2}>0 \\
& C\left(q, x_{1}\right) x_{2}=C\left(q, x_{2}\right) x_{1} \text { for all } x_{1}, x_{2} \in \mathbb{R}^{n} \\
& \|C(q, x)\| \leqslant \rho\|x\| \text { with } \rho>0 \text { for all } x \in \mathbb{R}^{n}
\end{aligned}
$$

$\left((2 \mathrm{a})\right.$ and $(2 \mathrm{c})$ are for a revolute joint robot). In addition, we make the assumption that $w_{0}$ belongs to $L_{2}[0, \infty)$.

Considering a trajectory tracking control law [NIC 93], the control torque $\Gamma$ is composed of two terms : the first one introduced to compensate partially the effect of the nonlinearities in the robot model (1) and the second one, called $\Gamma_{r}$, used for tracking, disturbance attenuation and stability purposes. Hence this control law is defined as

$$
\Gamma=H(q) \ddot{q}_{d}+C(q, \dot{q}) \dot{q}_{d}+F_{v} \dot{q}_{d}+G(q)+\Gamma_{r}
$$

where $q_{d}, \dot{q}_{d}$ and $\ddot{q}_{d}$ are the known and bounded vectors of the desired angular positions, the desired angular velocities and the desired angular accelerations respectively. In this paper, we assume that only the joint angular position vector $q$ is measured, then the joint angular velocity vector $\dot{q}$ in the control law (3) must be replaced by its "estimate" $\widehat{\dot{q}}$. Define 
and

$$
\tilde{q}=q-q_{d}
$$

$$
\dot{\tilde{q}}=\dot{q}-\dot{q}_{d}
$$

as the angular position tracking and angular velocity tracking errors respectively. Introduce the state vector $x$, the reference vector $x_{d}$, the "state estimate" vector $\hat{x}$, the reference vector and the measured output vector $y$ given by

and

$$
\begin{gathered}
x=\left[\begin{array}{c}
\tilde{q} \\
\dot{\tilde{q}}
\end{array}\right], \\
x_{d}=\left[\begin{array}{c}
q_{d} \\
\dot{q}_{d}
\end{array}\right], \\
\hat{x}=\left[\begin{array}{c}
\hat{q}-q_{d} \\
\hat{\dot{q}}-\dot{q}_{d}
\end{array}\right]
\end{gathered}
$$

$$
y=\tilde{q} .
$$

Then from equations (1) and (3) the following state-space model is obtained

$$
\begin{aligned}
& \dot{x}=A x+B_{1} w+B_{2} u+D\left(\alpha\left(x+x_{d}\right)-\Phi\left(\hat{x}+x_{d}, x_{d}, C_{2}\left(x+x_{d}\right)\right)\right) \\
& v=C_{1} x+D_{12} u \\
& y=C_{2} x
\end{aligned}
$$

where the vector $v$ is the controlled output. Then in (4), the control input vector $u$, the disturbance input vector $w$ and the nonlinear functions $\alpha\left(x+x_{d}\right)$ and $\Phi\left(\hat{x}+x_{d}, x_{d}, C_{2}\left(x+x_{d}\right)\right)$ are given by

$$
\begin{aligned}
& u=H^{-1}(q) \Gamma_{r} \\
& w=H^{-1}(q) w_{0} \\
& \alpha\left(x+x_{d}\right)=H^{-1}(q)\left(C(q, \dot{q}) \dot{q}+F_{v} \dot{q}\right) \\
& \Phi\left(\hat{x}+x_{d}, x_{d}, C_{2}\left(x+x_{d}\right)\right)=H^{-1}(q)\left(C(q, \widehat{\dot{q}}) \dot{q}_{d}+F_{v} \dot{q}_{d}\right)
\end{aligned}
$$

respectively. Note that $w$ belongs to $L_{2}[0, \infty)$ since $w_{0}$ belongs to $L_{2}[0, \infty)$ and the inverse of the inertia matrix $H^{-1}(q)$ is bounded (see (2a)). Matrices

$$
A=\left[\begin{array}{cc}
0 & I_{n} \\
0 & 0
\end{array}\right], B_{1}=B_{2}=\left[\begin{array}{c}
0 \\
I_{n}
\end{array}\right], D=\left[\begin{array}{c}
0 \\
-I_{n}
\end{array}\right] \text { and } C_{2}=\left[\begin{array}{ll}
I_{n} & 0
\end{array}\right]
$$

are of appropriate dimensions. The nonlinear functions $\alpha\left(x+x_{d}\right)$ and $\Phi\left(\hat{x}+x_{d}, x_{d}, C_{2}\left(x+x_{d}\right)\right)$ satisfy $\alpha(0)=0$ and $\Phi\left(0, x_{d}, C_{2}\left(x+x_{d}\right)\right)=0$ (see $\left.(2 \mathrm{c})\right) . \quad C_{1}$ and $D_{12}$ are given real constant matrices of appropriate dimensions. From (2a)-(2c), the following properties which are useful in the controller design can be deduced.

Property 1. [BER 93b, NIC 93] For any vectors $\xi_{1}=\left[\begin{array}{l}\zeta_{1} \\ \eta_{1}\end{array}\right]$ and $\xi_{2}=\left[\begin{array}{c}\zeta_{2} \\ \eta_{2}\end{array}\right]$, where $\zeta_{1}, \eta_{1}, \zeta_{2}$ and $\eta_{2} \in \mathbb{R}^{n}$, verifying $\left\|\eta_{1}\right\| \leqslant \eta_{\max }$ and $\left\|\eta_{2}\right\| \leqslant \eta_{\max }$, there exists some constant $\kappa>0$ such that

$$
\left\|\alpha\left(\xi_{1}\right)-\alpha\left(\xi_{2}\right)\right\| \leqslant \kappa\left\|\xi_{1}-\xi_{2}\right\| .
$$

Using relation $(2 \mathrm{~b})$, the constant $\kappa$ can be majorized as follows : $\kappa \leqslant 2 h_{1}^{-1}\left(\rho+\left\|F_{v}\right\|\right) \eta_{\max }$.

Property 2. For any vector $\xi=\left[\begin{array}{l}\zeta \\ \eta\end{array}\right]$, where $\zeta$ and $\eta \in \mathbb{R}^{n}$, verifying $\|\eta\| \leqslant \eta_{\max }$, we have

$$
\left\|H^{-1}(\zeta)\left(C(\zeta, \eta)+F_{v}\right)\right\| \leqslant h_{1}^{-1}\left(\rho+\left\|F_{v}\right\|\right) \eta_{\max }=\kappa_{1} .
$$

Property 3. For any vector $\xi=\left[\begin{array}{l}\zeta \\ \eta\end{array}\right]$, where $\zeta$ and $\eta \in \mathbb{R}^{n}$, verifying $\|\eta\| \leqslant \eta_{\max }$, we have

$$
\left\|H^{-1}(\zeta) C(\zeta, \eta)\right\| \leqslant h_{1}^{-1} \rho \eta_{\max }=\kappa_{2} .
$$


The order of the proposed controller is $m-p$, where $m=2 n$ and $p=n$ are the dimensions of the state $x$ and the measured output $y$, respectively. The reduced-order controller is given by

$$
\begin{aligned}
& \dot{z}=N z+L y+g\left(z, x_{d}, y\right)+F u \\
& \hat{x}=M z+E y \\
& u=-K \hat{x}
\end{aligned}
$$

$\left(z \in \mathbb{R}^{n}\right.$ and $\left.\hat{x} \in \mathbb{R}^{2 n}\right)$ under the two following constraints

$$
\begin{aligned}
& \operatorname{det}\left[\begin{array}{ll}
M & E
\end{array}\right] \neq 0 \\
& y=C_{2} \hat{x} .
\end{aligned}
$$

Matrices $N, L, F, M, E, K$ and the function $g\left(z, x_{d}, y\right)$ are computed to achieve the closed-loop stability and to attenuate the influence of the external disturbance $w$ on the controlled output $v$. Notice that this controller is not based on an observer since the convergence of $\hat{x}$ to $x$ is guaranteed only in closed-loop. From constraints (10a) and (10b), there exists a matrix $T$ such that

$$
\left[\begin{array}{ll}
M & E
\end{array}\right]\left[\begin{array}{c}
T \\
C_{2}
\end{array}\right]=\left[\begin{array}{c}
T \\
C_{2}
\end{array}\right]\left[\begin{array}{ll}
M & E
\end{array}\right]=I,
$$

then

$$
z=T \hat{x} .
$$

Therefore, with the following state variable

$$
X=\left[\begin{array}{c}
x \\
z-T x
\end{array}\right]=\left[\begin{array}{l}
x \\
e
\end{array}\right],
$$

and using relation (11), the closed-loop system is given by

$$
\begin{aligned}
& \dot{X}=\bar{A} X+\beta\left(x, \hat{x}, x_{d}\right)+\bar{B} w \\
& v=\bar{C} X
\end{aligned}
$$

where

$$
\begin{aligned}
& \bar{A}=\left[\begin{array}{ll}
\bar{A}_{11} & \bar{A}_{12} \\
\bar{A}_{21} & \bar{A}_{22}
\end{array}\right]=\left[\begin{array}{cc}
A-B_{2} K & -B_{2} K M \\
f(T) & \left(T B_{2}-F\right) K M+N
\end{array}\right] \\
& \bar{B}=\left[\begin{array}{l}
B_{1} \\
\bar{B}_{2}
\end{array}\right]=\left[\begin{array}{c}
B_{1} \\
-T B_{1}
\end{array}\right] \\
& \bar{C}=\left[\begin{array}{ll}
\bar{C}_{1} & \bar{C}_{2}
\end{array}\right]=\left[\begin{array}{cc}
C_{1}-D_{12} K & -D_{12} K M
\end{array}\right] \\
& \beta\left(x, \hat{x}, x_{d}\right)=\left[\begin{array}{c}
D\left(\alpha\left(x+x_{d}\right)-\Phi\left(\hat{x}+x_{d}, x_{d}, C_{2}\left(x+x_{d}\right)\right)\right) \\
g\left(z, x_{d}, y\right)-T D\left(\alpha\left(x+x_{d}\right)-\Phi\left(\hat{x}+x_{d}, x_{d}, C_{2}\left(x+x_{d}\right)\right)\right)
\end{array}\right] \\
& f(T)=-T\left(A-B_{2} K\right)+L C_{2}+N T-F K .
\end{aligned}
$$

Notice that the variable $z$ does not appear in the arguments of function $\beta$ since this variable can be directly deduced from $\hat{x}$ by using (12). For simplicity sake, $\hat{x}$ is using as argument of function $\beta$ in (14a), but from (10), (11) and (13), note that we have

$$
\hat{x}=M e+x .
$$

To evaluate the closed-loop disturbance attenuation between $w$ and $v$, we use the following definition of the $L_{2}$-gain given in [ISI 92] and [VDS 92].

Definition 1. Given a prescribed level of attenuation $\gamma>0$, the robot in closed-loop (14) is said to have $L_{2}$-gain less than or equal to $\gamma$ if

$$
\int_{0}^{\infty}\|v(t)\|^{2} d t \leqslant \gamma^{2} \int_{0}^{\infty}\|w(t)\|^{2} d t
$$

for all $w \in L_{2}[0, \infty)$ and with zero initial condition. The system (14) has $L_{2}$-gain less than $\gamma$ if there exists some $0 \leqslant \gamma_{0}<\gamma$ such that (17) holds for $\gamma_{0}$. 


\section{Reduced-Order Output Feedback Controller Design}

According to Definition 1, a systematic method is proposed to design the reduced-order controller (9) based on a direct extension of the well-known bounded real lemma, which is currently used in the $H_{\infty}$ linear control. From this approach, two algebraic Riccati inequalities are obtained from the $L_{2^{-}}$ gain constraint (17) of the closed-loop relation from $w$ to $v$. Then, for the rigid robot manipulator (4), sufficient conditions on the reduced-order output feedback controller (9) are given to guarantee the asymptotic stability of the closed-loop equilibrium point $X=0$ and to obtain a prescribed level for external disturbance attenuation with trajectory tracking.

\subsection{Preliminary Results}

As preliminary results, two lemmas are given : the first one concerns the parameterization of matrices $T, M$ and $E$ used in the controller design (see (9b) and (12)), the second one gives a sufficient condition to ensure the closed-loop $L_{2}$-gain objective (17). A useful parameterization of matrices $T, M$ and $E$ is given in Lemma 1.

Lemma 1. Let $C_{2}=\left[\begin{array}{ll}I_{n} & 0\end{array}\right]$. Matrices $T, M$ and $E$ given by

$$
\begin{aligned}
& T=R-\varphi C_{2}=\left[\begin{array}{ll}
R_{1}-\varphi & R_{2}
\end{array}\right] \\
& M=\left[\begin{array}{c}
0 \\
R_{2}^{-1}
\end{array}\right] \\
& E=\left[\begin{array}{c}
I \\
R_{2}^{-1}\left(\varphi-R_{1}\right)
\end{array}\right]
\end{aligned}
$$

are solutions to equation (11), where $\varphi$ and $R=\left[\begin{array}{ll}R_{1} & R_{2}\end{array}\right]$ are arbitrary matrices of appropriate dimensions such that $\left[\begin{array}{c}R \\ C_{2}\end{array}\right]$ is non-singular.

Proof. To parameterize the solutions of (11), let us introduce an arbitrary matrix $R$ such that $\left[\begin{array}{c}R \\ C_{2}\end{array}\right]$ is non-singular, with $R=\left[\begin{array}{ll}R_{1} & R_{2}\end{array}\right]$. Since $C_{2}=\left[\begin{array}{ll}I_{n} & 0\end{array}\right]$, the regularity condition is reduced to $\operatorname{det} R_{2} \neq 0$. In order to satisfy the constraint (11), matrix $T$ can be chosen as follows

$$
\left[\begin{array}{c}
T \\
C_{2}
\end{array}\right]=\left[\begin{array}{cc}
I & -\varphi \\
0 & I
\end{array}\right]\left[\begin{array}{c}
R \\
C_{2}
\end{array}\right]
$$

where $\varphi$ is an arbitrary matrix of appropriate dimension. Hence, since $C_{2}=\left[\begin{array}{ll}I_{n} & 0\end{array}\right]$, matrix $T$ is given by (18a). From (11) and (18a), it is easy to show that $M$ and $E$ are given by (18b) and (18c) respectively.

Note that, by using Lemma 1, inserting (18b) into (16) gives

$$
\widehat{\dot{q}}=R_{2}^{-1} e+\dot{q} .
$$

Sufficient conditions to obtain a closed-loop $L_{2}$-gain objective less than or equal to $\gamma$, between the external disturbance $w$ and the controlled output $v$, are given in the following lemma.

Lemma 2. Assume that relations (18a)-(18c) and Properties 1, 2 and 3 are satisfied. According to Definition 1, if the following relations hold

$$
g\left(z, x_{d}, y\right)=T D\left(\alpha\left(\hat{x}+x_{d}\right)-\Phi\left(\hat{x}+x_{d}, x_{d}, C_{2}\left(x+x_{d}\right)\right)\right)
$$

and

$$
\left[\begin{array}{cc}
\bar{A}_{11}^{T} & \bar{A}_{21}^{T} \\
\bar{A}_{12}^{T} & \bar{A}_{22}^{T}
\end{array}\right]\left[\begin{array}{cc}
P_{1} & 0 \\
0 & P_{2}
\end{array}\right]+\left[\begin{array}{cc}
P_{1} & 0 \\
0 & P_{2}
\end{array}\right]\left[\begin{array}{ll}
\bar{A}_{11} & \bar{A}_{12} \\
\bar{A}_{21} & \bar{A}_{22}
\end{array}\right]+\left[\begin{array}{ccc}
0 & 0 & 0 \\
0 & \left(\kappa_{1}^{2}+\mu^{2}\right) I_{n} & 0 \\
0 & 0 & \left(\kappa^{2}+\kappa_{2}^{2}+\frac{\kappa_{1}^{2} \kappa_{2}^{2}}{\mu^{2}}\right) M M^{T}
\end{array}\right]
$$




$$
+\left[\begin{array}{cc}
\bar{C}_{1}^{T} \bar{C}_{1} & \bar{C}_{1}^{T} \bar{C}_{2} \\
\bar{C}_{2}^{T} \bar{C}_{1} & \bar{C}_{2}^{T} \bar{C}_{2}
\end{array}\right]+\left[\begin{array}{cc}
P_{1} & 0 \\
0 & P_{2}
\end{array}\right]\left[\begin{array}{cc}
\gamma^{-2} \bar{B}_{1} \bar{B}_{1}^{T}+D D^{T} & \gamma^{-2} \bar{B}_{1} \bar{B}_{2}^{T} \\
\gamma^{-2} \bar{B}_{2} \bar{B}_{1}^{T} & \left(\gamma^{-2}+1\right) R_{2} R_{2}^{T}
\end{array}\right]\left[\begin{array}{cc}
P_{1} & 0 \\
0 & P_{2}
\end{array}\right]=\left[\begin{array}{cc}
Q_{11} & Q_{12} \\
Q_{12}^{T} & Q_{22}
\end{array}\right]<0
$$

where

$$
P=P^{T}=\left[\begin{array}{cc}
P_{1} & 0 \\
0 & P_{2}
\end{array}\right]>0
$$

and $\mu$ is an arbitrary positive scalar, then the closed-loop system (14) has a $L_{2}$-gain less than or equal to $\gamma$ between the external disturbance $w$ and the controlled output $v$.

Proof. Consider the closed-loop representation (14). For $P=P^{T}>0$, let us introduce the following performance measure with an initial state corresponding to the equilibrium $(X=0)$

$$
\begin{aligned}
& J=J_{1}-X^{T}(\infty) P X(\infty) \\
& J_{1}=\int_{0}^{\infty}\left\{v^{T} v-\gamma^{2} w^{T} w+\frac{d}{d t}\left(X^{T} P X\right)\right\} d t .
\end{aligned}
$$

According to [VDS 92], the performance index $\mathrm{J}$ (23a) is an integral dissipation inequality. Note that condition (17) is equivalent to $J \leqslant 0$. Using equation (14b), $J_{1}$ can be written as

$$
J_{1}=\int_{0}^{\infty}\left\{X^{T} \bar{C}^{T} \bar{C} X-\gamma^{2} w^{T} w+\dot{X}^{T} P X+X^{T} P \dot{X}\right\} d t
$$

Combining (14) and (24) yields

$$
\begin{aligned}
J_{1}=\int_{0}^{\infty}\left\{X ^ { T } \left(\bar{A}^{T} P+P \bar{A}+\right.\right. & \left.\bar{C}^{T} \bar{C}+\gamma^{-2} P \bar{B} \bar{B}^{T} P\right) X+\beta^{T}\left(x, \hat{x}, x_{d}\right) P X \\
& \left.+X^{T} P \beta\left(x, \hat{x}, x_{d}\right)-\left(w-\gamma^{-2} \bar{B}^{T} P X\right)^{T} \gamma^{2}\left(w-\gamma^{-2} \bar{B}^{T} P X\right)\right\} d t .
\end{aligned}
$$

Using (15d), (21) and (22b), we obtain

$$
\begin{aligned}
& \beta^{T}\left(x, \hat{x}, x_{d}\right) P X+X^{T} P \beta\left(x, \hat{x}, x_{d}\right)=\left\|\theta\left(x, \hat{x}, x_{d}\right)\right\|^{2}-\left\|\theta\left(x, \hat{x}, x_{d}\right)-D^{T} P_{1} x\right\|^{2}+\left\|D^{T} P_{1} x\right\|^{2} \\
& -\left\|\alpha\left(\hat{x}+x_{d}\right)-\alpha\left(x+x_{d}\right)-D^{T} T^{T} P_{2} e\right\|^{2}+\left\|\alpha\left(\hat{x}+x_{d}\right)-\alpha\left(x+x_{d}\right)\right\|^{2}+\left\|D^{T} T^{T} P_{2} e\right\|^{2}
\end{aligned}
$$

where

$$
\begin{aligned}
\theta\left(x, \hat{x}, x_{d}\right) & =\alpha\left(x+x_{d}\right)-\Phi\left(\hat{x}+x_{d}, x_{d}, C_{2}\left(x+x_{d}\right)\right) \\
& =H^{-1}(q) C(q, \dot{q}) \dot{q}-H^{-1}(q) C(q, \widehat{\dot{q}}) \dot{q}_{d}+H^{-1}(q) F_{v} \dot{\tilde{q}} .
\end{aligned}
$$

At this step of the proof, it is interesting to show that the function $\theta\left(x, \hat{x}, x_{d}\right)$ has an affine dependence on variables $\dot{\tilde{q}}$ and $e$, namely an affine dependence on the closed-loop state variable $X$ (13). By using property $(2 \mathrm{~b})$ and by subtracting and adding the same term, $\theta\left(x, \hat{x}, x_{d}\right)$ in $(27)$ can be written as

$$
\theta\left(x, \hat{x}, x_{d}\right)=H^{-1}(q)\left(C(q, \dot{q}) \dot{q}-C(q, \dot{q}) \dot{q}_{d}+C\left(q, \dot{q}_{d}\right) \dot{q}-C\left(q, \dot{q}_{d}\right) \widehat{\dot{q}}+F_{v} \dot{\tilde{q}}\right)
$$

or equivalently

$$
\theta\left(x, \hat{x}, x_{d}\right)=H^{-1}(q) C(q, \dot{q})\left(\dot{q}-\dot{q}_{d}\right)-H^{-1}(q) C\left(q, \dot{q}_{d}\right)(\widehat{\dot{q}}-\dot{q})+H^{-1}(q) F_{v} \dot{\tilde{q}} .
$$

By inserting (20) into (29), the function $\theta\left(x, \hat{x}, x_{d}\right)$ has an affine dependence on variables $\dot{\tilde{q}}$ and $e$

$$
\theta\left(x, \hat{x}, x_{d}\right)=H^{-1}(q)\left(C(q, \dot{q})+F_{v}\right) \dot{\tilde{q}}-H^{-1}(q) C\left(q, \dot{q}_{d}\right) R_{2}^{-1} e .
$$

Using Properties 1, 2 and 3 (see (6), (7) and (8)), and combining (16), (18b), (25), (26) and (30), the following inequality is obtained by completing the squares 


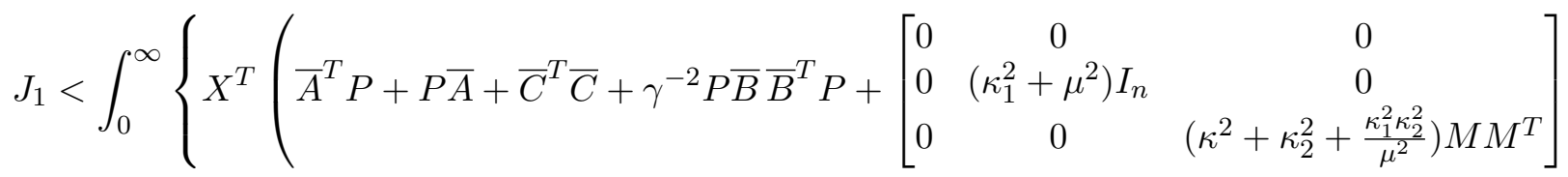

$$
\begin{aligned}
& \left.+\left[\begin{array}{cc}
P_{1} & 0 \\
0 & P_{2}
\end{array}\right]\left[\begin{array}{cc}
D D^{T} & 0 \\
0 & T D D^{T} T^{T}
\end{array}\right]\left[\begin{array}{cc}
P_{1} & 0 \\
0 & P_{2}
\end{array}\right]\right) X-\left\|\theta\left(x, \hat{x}, x_{d}\right)-D^{T} P_{1} x\right\|^{2} \\
& \left.-\gamma^{2}\left\|w-\gamma^{-2} \bar{B}^{T} P X\right\|^{2}-\left\|\alpha\left(\hat{x}+x_{d}\right)-\alpha\left(x+x_{d}\right)-D^{T} T^{T} P_{2} e\right\|^{2}\right\} d t
\end{aligned}
$$

where $\mu>0$ is arbitrary. Notice that the optimal value of $\mu>0$ minimizing

$$
\bar{\kappa}=\kappa_{1}^{2}+\mu^{2}+\kappa^{2}+\kappa_{2}^{2}+\frac{\kappa_{1}^{2} \kappa_{2}^{2}}{\mu^{2}}
$$

reduces the conservatism in inequality (31). This value is given by

$$
\mu_{\mathrm{opt}}=\sqrt{\kappa_{1} \kappa_{2}}
$$

From $\bar{B}_{1}=B_{1}=\left[\begin{array}{c}0 \\ I_{n}\end{array}\right], D=\left[\begin{array}{c}0 \\ -I_{n}\end{array}\right]$, and $T$ defined by (18a), expressions $T D D^{T} T^{T}$ and $\bar{B}_{2} \bar{B}_{2}^{T}$ in the inequality (31) become

$$
\begin{aligned}
& T D D^{T} T^{T}=R_{2} R_{2}^{T} \\
& \bar{B}_{2} \bar{B}_{2}^{T}=T B_{1} B_{1}^{T} T^{T}=R_{2} R_{2}^{T} .
\end{aligned}
$$

Using the fact that $X^{T}(\infty) P X(\infty) \geqslant 0$, the $L_{2}$-gain attenuation (17) is satisfied if the performance index $J_{1}(25)$ is negative. From (31) this can be achieved if the algebraic Riccati inequality (22a) holds.

\subsection{Main Result}

Based on Lemma 2, the sufficient conditions to obtain an external disturbance attenuation in a $L_{2}$-gain sense with trajectory tracking and asymptotic stability via the reduced-order output feedback controller (9) are given in Theorem 1. The construction of this controller is given in Lemma 3.

Theorem 1. Assume that relations (18a)-(18c), (21) and (22a) are satisfied. If we choose

$$
\begin{aligned}
& \left\|\dot{q}_{d}\right\| \leqslant V_{M} \\
& X(0) \in \mathbb{D}=\left\{X / V(X) \leqslant V_{\max }\right\}
\end{aligned}
$$

with

$$
\begin{aligned}
& V(X)=X^{T} P X \\
& V_{\max }=\min \left(\lambda_{m}\left(P_{1}\right) \delta_{v}^{2}, \lambda_{m}\left(P_{2}\right) \delta_{e}^{2}\right)
\end{aligned}
$$

where $\delta_{v}$ and $\delta_{e}$ are given positive scalars, $V_{M}$ is the maximum desired velocity, and $\lambda_{m}(\cdot)$ denotes the minimum eigenvalue of the corresponding matrix, then the controller (9) of order $m-p$ ensures an asymptotic stability of the equilibrium point $(\tilde{q}, \dot{\tilde{q}}, e)=0$ of the robot system in closed-loop (14) for all initial state into the domain $\mathbb{D}$, and yields an attenuation of the disturbance input according to Definition 1.

Proof. To analyze the asymptotic stability of the equilibrium point $(\tilde{q}, \dot{\tilde{q}}, e)=0$ of the closed-loop robot system (14) without perturbation $(w(t) \equiv 0)$, consider the Lyapunov function candidate $V(X)$ defined in (32a) where the matrix $P=P^{T}>0$, given by $(22 \mathrm{~b})$, is a solution of the algebraic Riccati inequality (22a). The time derivative of the Lyapunov function $V(X)$ (36a) along the dynamics (14a) with $w(t) \equiv 0$ can be expressed as

$$
\dot{V}(X(t))=X^{T}\left(\bar{A}^{T} P+P \bar{A}\right) X+\beta^{T}\left(x, \hat{x}, x_{d}\right) P X+X^{T} P \beta\left(x, \hat{x}, x_{d}\right) .
$$


Then according to relations (26)-(30), the following inequality is obtained

$$
\begin{aligned}
\dot{V}(X(t)) \leqslant X^{T}\left(\bar{A}^{T} P+P \bar{A}+\left[\begin{array}{cc}
P_{1} & 0 \\
0 & P_{2}
\end{array}\right]\left[\begin{array}{cc}
D D^{T} & 0 \\
0 & T D D^{T} T^{T}
\end{array}\right]\left[\begin{array}{cc}
P_{1} & 0 \\
0 & P_{2}
\end{array}\right]\right. \\
\left.+\left[\begin{array}{ccc}
0 & 0 & 0 \\
0 & \left(\kappa_{1}^{2}+\mu^{2}\right) I_{n} & 0 \\
0 & 0 & \left(\kappa^{2}+\kappa_{2}^{2}+\frac{\kappa_{1}^{2} \kappa_{2}^{2}}{\mu^{2}}\right) M M^{T}
\end{array}\right]\right) X .
\end{aligned}
$$

From the algebraic Riccati inequality (22a) given in Lemma 2, it is deduced that $\dot{V}(X(t))<0$ for $X=0$.

The use of scalars $\kappa, \kappa_{1}$ and $\kappa_{2}$ require that the bounds of $\dot{q}$ and $\widehat{\dot{q}}$ are known (see Properties 1,2 and 3). Thank to the definition of the domain $\mathbb{D}(35)$ these bounds can be computed as follows. The Lyapunov function (36a) is a decreasing function of time. Then, by using (22b), (35b) and (36a), the following inequality holds

$$
\lambda_{m}\left(P_{1}\right)\|\dot{\tilde{q}}\|^{2}+\lambda_{m}\left(P_{2}\right)\|e\|^{2} \leqslant V(X(t)) \leqslant V(X(0)) \leqslant V_{\max } .
$$

Since $V_{\max }$ is given by $(36 \mathrm{a})$, then

$$
\begin{aligned}
& \|\dot{\tilde{q}}\|^{2} \leqslant \frac{V_{\max }}{\lambda_{m}\left(P_{1}\right)} \leqslant \delta_{v}^{2} \\
& \|e\|^{2} \leqslant \frac{V_{\max }}{\lambda_{m}\left(P_{2}\right)} \leqslant \delta_{e}^{2} .
\end{aligned}
$$

From $\dot{q}=\dot{\tilde{q}}+\dot{q}_{d},(20),(35 \mathrm{a})$ and (40) we have

$$
\begin{aligned}
& \|\dot{q}\| \leqslant\|\dot{\tilde{q}}\|+\left\|\dot{q}_{d}\right\| \leqslant \delta_{v}+V_{M} \\
& \|\hat{\dot{q}}\| \leqslant\left\|R_{2}^{-1}\right\|\|e\|+\|\dot{q}\| \leqslant\left\|R_{2}^{-1}\right\| \delta_{e}+\delta_{v}+V_{M} .
\end{aligned}
$$

Then Properties 1, 2 and 3 hold with

$$
\begin{aligned}
& \kappa=\max _{\xi \in \Xi}\left\|\frac{\partial \alpha}{\partial \xi}\right\| \\
& \kappa_{1}=h_{1}^{-1} \rho\left(\delta_{v}+V_{M}\right) \\
& \kappa_{2}=h_{1}^{-1} \rho V_{M}
\end{aligned}
$$

where

$$
\Xi=\left\{\xi / \xi=\left[\begin{array}{l}
\zeta \\
\eta
\end{array}\right], \zeta \in \mathbb{R}^{n}, \eta \in \mathbb{R}^{n} \text { and }\|\eta\| \leqslant\left(\left\|R_{2}^{-1}\right\| \delta_{e}+\delta_{v}+V_{M}\right)\right\}
$$

The use of the Theorem 1 for the controller design requires to solve the algebraic Riccati inequality (22a). This is the aim of the following lemma.

Lemma 3. The algebraic Riccati inequality (22a) with $Q_{12}=0$ holds if the following three conditions are verified.

(i) There exists a symmetric and positive definite matrix $P_{1}$ satisfying the following algebraic Riccati inequality

$$
\begin{aligned}
(A- & \left.B_{2}\left(D_{12}^{T} D_{12}\right)^{-1} D_{12}^{T} C_{1}\right)^{T} P_{1}+P_{1}\left(A-B_{2}\left(D_{12}^{T} D_{12}\right)^{-1} D_{12}^{T} C_{1}\right)+\left(\kappa_{1}^{2}+\mu^{2}\right)\left[\begin{array}{cc}
0 & 0 \\
0 & I_{n}
\end{array}\right] \\
& +C_{1}^{T}\left(I-D_{12}\left(D_{12}^{T} D_{12}\right)^{-1} D_{12}^{T}\right) C_{1}+P_{1}\left(\gamma^{-2} B_{1} B_{1}^{T}+D D^{T}-B_{2}\left(D_{12}^{T} D_{12}\right)^{-1} B_{2}^{T}\right) P_{1}<0
\end{aligned}
$$


(ii) There exist a symmetric and positive definite matrix $Q_{2}=P_{2}^{-1}$ and a scaling parameter $\varepsilon>0$ satisfying the following algebraic Riccati inequality

$$
\begin{aligned}
Q_{2}\left(M ^ { T } \left(\left(P_{1} B_{2}+C_{1}^{T} D_{12}\right)\left(D_{12}^{T} D_{12}\right)^{-1}(\right.\right. & \left.\left.B_{2}^{T} P_{1}+D_{12}^{T} C_{1}\right)+\left(\kappa^{2}+\kappa_{2}^{2}+\frac{\kappa_{1}^{2} \kappa_{2}^{2}}{\mu^{2}}\right)\right) M \\
& \left.-\frac{\Omega^{T} \Omega}{\varepsilon}\right) Q_{2}+Q_{2} \Psi^{T}+\Psi Q_{2}+\left(\gamma^{-2}+1\right) R_{2} R_{2}^{T}<0
\end{aligned}
$$

where

$$
\begin{aligned}
& \Psi=R A_{\gamma} M \\
& \Omega=C_{2} A_{\gamma} M \\
& A_{\gamma}=A+\gamma^{-2} B_{1} B_{1}^{T} P_{1}
\end{aligned}
$$

(iii) The controller gains $K$ and $\varphi$, and controller matrices $N, L$ and $F$, are given by

$$
\begin{aligned}
& K=\left(D_{12}^{T} D_{12}\right)^{-1}\left(B_{2}^{T} P_{1}+D_{12}^{T} C_{1}\right) \\
& \varphi=\frac{1}{2 \varepsilon} Q_{2} \Omega^{T} \\
& N=T A_{\gamma} M-Z K M \\
& L=T A_{\gamma} E-Z K E \\
& F=T B_{2}-Z
\end{aligned}
$$

where $Z$ is an arbitrary matrix of appropriate dimension.

Proof. The algebraic Riccati inequality (22a) holds if the following relations are satisfied

$$
\begin{aligned}
Q_{11} & =\bar{A}_{11}^{T} P_{1}+P_{1} \bar{A}_{11}+P_{1}\left(\gamma^{-2} \bar{B}_{1} \bar{B}_{1}^{T}+D D^{T}\right) P_{1}+\bar{C}_{1}^{T} \bar{C}_{1}+\left(\kappa_{1}^{2}+\mu^{2}\right)\left[\begin{array}{cc}
0 & 0 \\
0 & I_{n}
\end{array}\right]<0 \\
Q_{12} & =\bar{A}_{21}^{T} P_{2}+P_{1} \bar{A}_{12}+\gamma^{-2} P_{1} \bar{B}_{1} \bar{B}_{2}^{T} P_{2}+\bar{C}_{1}^{T} \bar{C}_{2}=0 \\
Q_{22} & =\bar{A}_{22}^{T} P_{2}+P_{2} \bar{A}_{22}+\left(\gamma^{-2}+1\right) P_{2} R_{2} R_{2}^{T} P_{2}+\left(\kappa^{2}+\kappa_{2}^{2}+\frac{\kappa_{1}^{2} \kappa_{2}^{2}}{\mu^{2}}\right) M^{T} M+\bar{C}_{2}^{T} \bar{C}_{2}<0 .
\end{aligned}
$$

Note that the algebraic Riccati inequalities (48a) and (48c) require the stability of $\bar{A}_{11}$ and $\bar{A}_{22}$.

Substituting the expressions of $\bar{A}_{11}, \bar{B}_{1}$, and $\bar{C}_{1}$ defined by (15a)-(15c) into the algebraic Riccati inequality (48a), the following inequality is obtained

$$
\begin{aligned}
\left(A-B_{2} K\right)^{T} P_{1}+P_{1}\left(A-B_{2} K\right)+P_{1}\left(\gamma^{-2} B_{1} B_{1}^{T}+D D^{T}\right) P_{1} & \\
& +\left(C_{1}-D_{12} K\right)^{T}\left(C_{1}-D_{12} K\right)+\left(\kappa_{1}^{2}+\mu^{2}\right)\left[\begin{array}{cc}
0 & 0 \\
0 & I_{n}
\end{array}\right]<0 .
\end{aligned}
$$

Inserting the gain matrix $K$ (47a) into (49), then the algebraic Riccati inequality (44) is obtained. Then it follows that the matrix $\bar{A}_{11}=A-B_{2} K$ is stable if condition (i) is achieved.

By using (46c) and (47e), inserting the expressions (15a)-(15c) and (15e) into (48b) yields

$$
P_{2}\left(N T+L C_{2}+Z K-T A_{\gamma}\right)-M^{T} K^{T}\left(B_{2}^{T} P_{1}+D_{12}^{T} C_{1}-D_{12}^{T} D_{12} K\right)=0 .
$$

From the expression of $K$ (47a), we can remark that

$$
B_{2}^{T} P_{1}+D_{12}^{T} C_{1}-D_{12}^{T} D_{12} K=0 .
$$

Since $P_{2}>0$, inserting (51) into (50) yields

$$
N T+L C_{2}+Z K-T A_{\gamma}=0 .
$$


Hence, multiplying (52) by the non-singular matrix $\left[\begin{array}{ll}M & E\end{array}\right]$ and using the relation (11), equations $(47 \mathrm{c})$ and $(47 \mathrm{~d})$ are obtained.

By using (47e), matrix $\bar{A}_{22}$ can be rewritten as

$$
\bar{A}_{22}=N+Z K M
$$

Using the expressions of $N$ and $T$ given by (47c) and (18a) respectively, the matrix $\bar{A}_{22}$ in (48) becomes

$$
\bar{A}_{22}=\Psi-\varphi \Omega
$$

where $\Psi$ and $\Omega$ are given by (47a) and (47c) respectively.

By pre and post-multiplying the algebraic Riccati inequality (48c) by $Q_{2}=P_{2}^{-1}$, and by using (15c) and (54), an equivalent inequality is obtained

$$
\begin{aligned}
Q_{2}\left(M^{T} K^{T} D_{12}^{T} D_{12} K M+\left(\kappa^{2}+\kappa_{2}^{2}+\right.\right. & \left.\left.\frac{\kappa_{1}^{2} \kappa_{2}^{2}}{\mu^{2}}\right) M^{T} M\right) Q_{2} \\
& +Q_{2}(\Psi-\varphi \Omega)^{T}+(\Psi-\varphi \Omega) Q_{2}+\left(\gamma^{-2}+1\right) R_{2} R_{2}^{T}<0 .
\end{aligned}
$$

Choosing the gain matrix $\varphi$ given by (47b), then the algebraic Riccati inequality (45) is obtained. Then it follows that $\bar{A}_{22}$ is a stable matrix if condition (ii) is achieved. This ends the proof of the lemma.

From (54), to obtain a matrix $\bar{A}_{22}$ stable, the pair $(\Omega, \Psi)$ must be detectable. The following lemma gives the necessary and sufficient condition to obtain a detectable pair $(\Omega, \Psi)$.

Lemma 4. The unobservable modes of the two pairs $\left(C_{2}, A_{\gamma}\right)$ and $(\Omega, \Psi)$ are the same.

Proof. Let us introduce the following unimodular matrices

$$
U_{1}=\left[\begin{array}{cc}
R & -s \varphi+R A_{\gamma} E \\
C_{2} & -s I+C_{2} A_{\gamma} E \\
0 & I
\end{array}\right] \text { and } U_{2}=\left[\begin{array}{ll}
M & E
\end{array}\right] .
$$

Then, it follows that

$$
\begin{aligned}
\operatorname{rank}\left[\begin{array}{c}
s I-A_{\gamma} \\
C_{2}
\end{array}\right] & =\operatorname{rank} U_{1}\left[\begin{array}{c}
s I-A_{\gamma} \\
C_{2}
\end{array}\right] U_{2} \\
& =\operatorname{rank}\left[\begin{array}{cc}
s I-\Psi & 0 \\
\Omega & 0 \\
0 & I
\end{array}\right] .
\end{aligned}
$$

This completes the proof of the lemma.

Note that the pair $\left(C_{2}, A_{\gamma}\right)$ is observable, then the Lemma 4 implies that the pair $(\Omega, \Psi)$ is observable. In addition, since the pair $\left(A, B_{2}\right)$ is reachable, the two algebraic Riccati inequalities in Lemma 3 are solvable (see Remarks 2 and 3 ).

Remark 1 The proposed reduced-order controller requires to solve two algebraic Riccati inequalities (44) and (45) which are not mutually-coupled as in [DOY 89] and [SAM 90], but unilaterally-coupled as in [HSU 94]. Unlike in [HSU 94], the matrix $N$ (47c) is not obtained by solving an eigenvalue/eigenvector problem by using a Jordan decomposition : the parameterization (18a) of matrix $T$ allows to overcome this difficulty and the computation of matrix $N$ is reduced to the choice of an arbitrary matrix $Z$ (see (47c)). Then, our approach allows to have $T B_{2}=F$ and consequently $\bar{A}_{22}=N$ by choosing $Z=0$. This is not the case in [HSU 94] where $F$ must be different from $T B_{2}$. 
Remark 2 Note that there are no Hamilton-Jacobi equations to be solved as in [VDS 92], [ISI 92] and [KRE 94]. These equations are hard to solve exactly, and the size of the neighborhood for a local solution based on a linearization technique is often undetermined [VDS 92]. For the proposed controller, the use of an extension of the bounded real lemma to a non linear system satisfying a semi-global Lipschitz condition (6) leads to algebraic Riccati inequalities which can be easily solved as linear matrix inequalities by using convex semi-definite programming [BOY 94], [EGH 95], [GAH 95] or by transforming the algebraic Riccati inequalities into algebraic Riccati equations.

Remark 3 Consider the algebraic Riccati inequalities (44) and (45) in Lemma 3 and define the two following matrices

$$
W_{1}=\gamma^{-2} B_{1} B_{1}^{T}+D D^{T}-B_{2}\left(D_{12}^{T} D_{12}\right)^{-1} B_{2}^{T}
$$

and

$$
\begin{aligned}
W_{2}=M^{T}\left(P_{1} B_{2}+C_{1}^{T} D_{12}\right)\left(D_{12}^{T} D_{12}\right)^{-1}\left(B_{2}^{T} P_{1}+D_{12}^{T} C_{1}\right) & M \\
& +\left(\kappa^{2}+\kappa_{2}^{2}+\frac{\kappa_{1}^{2} \kappa_{2}^{2}}{\mu^{2}}\right) M^{T} M-\frac{\Omega^{T} \Omega}{\varepsilon} .
\end{aligned}
$$

From (59), for decreasing $\varepsilon, W_{2}$ decreases in a semi-positive definite sense. Then for a given $\gamma$, algebraic Riccati inequality (45) cannot be solved when $\varepsilon$ is close to zero, the value of $\gamma$ must be increased. Then $\varepsilon$ is a useful design parameter to solve (45) for a given disturbance attenuation $\gamma$ (see the step 9 in the design procedure). Similarly, if matrix $D_{12}$ in the objective equation (4b) is replaced by $\nu D_{12}$ with $\nu>0$, then $W_{1}$ in (58) decreases in a semi-positive definite sense when $\nu$ increases. One can see that $\frac{1}{\nu}$ plays a similar role in (58) as $\varepsilon$ in (59). Since matrix $W_{3}$ given by

$$
W_{3}=\left(\kappa_{1}^{2}+\mu^{2}\right)\left[\begin{array}{cc}
0 & 0 \\
0 & I_{n}
\end{array}\right]+C_{1}^{T}\left(I-D_{12}\left(D_{12}^{T} D_{12}\right)^{-1} D_{12}^{T}\right) C_{1}
$$

does not change when $\nu$ is modified, then $\nu$ can be used as a design parameter to solve the algebraic Riccati inequality (44) for a given disturbance attenuation $\gamma$ (see the step 5 in the design procedure).

Remark 4 If we put $\dot{q}_{d} \equiv 0$, the proposed trajectory tracking controller becomes a point-to-point controller with gravity compensation (see [NIC 93] and [LEW 93]). In this case, the control law $\Gamma$ in (3) becomes

$$
\Gamma=G(q)+\Gamma_{r}
$$

\section{Reduced-Order Output Feedback Controller Design Procedure}

The design procedure of the reduced-order output feedback controller (9) can be made as follows.

Step 1 Choose the scalars $V_{M}>0, \delta_{v}>0, \delta_{e}>0$ and an arbitrary matrix $R$ such that $\operatorname{det}\left[\begin{array}{c}R \\ C_{2}\end{array}\right] \neq 0$ with $R=\left[\begin{array}{ll}R_{1} & R_{2}\end{array}\right]$.

Step 2 Compute the Lipschitz constant $\kappa$ and the scalars $\kappa_{1}$ and $\kappa_{2}$ given by (42a), (42b) and (42c) respectively, and compute $\mu=\mu_{\mathrm{opt}}$ by (33).

Step 3 Choose a level of external disturbance attenuation $\gamma>0$.

Step 4 Solve the algebraic Riccati inequality (44).

Step 5 Check if $P_{1}>0$, then go to step 6, else, first, multiply $D_{12}$ by $\nu$, increase $\nu$ and go to step 4 or, second, increase $\gamma$ and go to step 4 .

Step 6 The controller gain $K$ and the matrix $A_{\gamma}$ are given by (47a) and (46c) respectively.

Step 7 The matrices $M, \Psi$ and $\Omega$ are given by (18b), (46a) and (46a) respectively, and choose $\varepsilon>0$.

Step 8 Solve the algebraic Riccati inequality (45).

Step 9 Check if $Q_{2}>0$, then go to step 10, else, first, decrease $\varepsilon$ and go to step 8 or, second, increase $\gamma$ and go to step 4 . 
Step 10 The gain matrix $\varphi$ is given by (47b).

Step 11 The matrices $T$ and $E$ are given by (18a) and (18c) respectively.

Step 12 After choosing an arbitrary matrix $Z$, the matrices $N, L$ and $F$ are given by (47c), (47d) and (47e) respectively.

Step 13 The function $g\left(z, x_{d}, y\right)$ is given by (21) with $\alpha\left(\hat{x}+x_{d}\right)$ and $\Phi\left(\hat{x}+x_{d}, x_{d}, y\right)$ given by (5c) and (5d) respectively.

Step 14 The domain is $\mathbb{D}=\left\{X / V(X) \leqslant V_{\max }\right\}$, where $P=\left[\begin{array}{cc}P_{1} & 0 \\ 0 & Q_{2}^{-1}\end{array}\right], V=X^{T} P X,\left\|\dot{q}_{d}\right\| \leqslant V_{M}$ and $V_{\max }$ is given by (36b).

The previous design procedure requires only off-line computations. These off-line computations does not include numerical difficulties since matrices $A, B_{1}, B_{2}, D$ and $C_{2}$ defined in section 2 are decoupled link by link. The on-line computations are reduced to the resolution of the differential equation (9a) and to the numerical evaluation of equations (3), (9b), (9c) and the numerical evaluation of the vector function $g\left(z, x_{d}, y\right)$ (see (21)) as in the design procedures presented in the literature ([BER 93b], [CAN 92], [KHE 96], [NIC 93]).

\section{Simulation Example}

To show the feasibility of the proposed design method, a simulation example is presented. Consider the 2-D.O.F rigid robot manipulator used by Berghuis and Nijmeijer [BER 93b], represented by figure 1 in the appendix. The robot matrices are characterized by

$$
\begin{gathered}
H(q)=\left[\begin{array}{cc}
9.77+2.02 \cos \left(q_{2}\right) & 1.26+1.01 \cos \left(q_{2}\right) \\
1.26+1.01 \cos \left(q_{2}\right) & 1.12
\end{array}\right], C(q, \dot{q})=\left[\begin{array}{cc}
-1.01 \sin \left(q_{2}\right) \dot{q}_{2} & 1.01 \sin \left(q_{2}\right)\left(\dot{q}_{1}+\dot{q}_{2}\right) \\
1.01 \sin \left(q_{2}\right) \dot{q}_{1} & 0
\end{array}\right] \text { and } \\
G(q)=\left[\begin{array}{c}
8.1 \sin \left(q_{1}\right)+1.13 \sin \left(q_{1}+q_{2}\right) \\
1.13 \sin \left(q_{1}+q_{2}\right)
\end{array}\right] .
\end{gathered}
$$

By using majorations, it can be seen that [BER 93b] (see (2a) and (2c))

$$
h_{1}=1 \mathrm{~kg} \mathrm{~m}^{-1}, h_{2}=25 \mathrm{~kg} \mathrm{~m}^{-1} \text { and } \rho=6 \mathrm{~kg} \mathrm{~m}^{2} \mathrm{~s}^{-1} .
$$

The parameters of the domain $\mathbb{D}$ are chosen as

$$
V_{M}=0.9 \mathrm{rad} \mathrm{s}^{-1}, \delta_{v}=0.45 \mathrm{rad} \mathrm{s}^{-1} \text { and } \delta_{e}=0.45 \mathrm{rad} \mathrm{s}^{-1} .
$$

Using relations (2b) and (42a)-(42c), the scalars $\kappa, \kappa_{1}$ and $\kappa_{2}$ are given by

$$
\kappa=16.2 \mathrm{~s}^{-1}, \kappa_{1}=8.1 \mathrm{~s}^{-1} \text { and } \kappa_{2}=5.4 \mathrm{~s}^{-1} \text {. }
$$

The design parameters are chosen as

$$
R_{1}=0, R_{2}=I_{2}, C_{2}=\left[\begin{array}{ll}
I_{2} & 0
\end{array}\right], D_{12}=I_{2}, Z=0, \gamma=2, \nu=0.4 \text { (see Remark 3) and } \varepsilon=0.0009 .
$$

In order to transform the algebraic Riccati inequalities (44) and (45) into algebraic Riccati equalities, solved with the "Robust Control Toolbox" of the software "Matlab", the matrices $350 \times I_{4}$ and $I_{2}$ have been added to the left hand side of inequalities (44) and (45) respectively.

From the previous data, the matrices of the controller (9) and (12) and the bound $V_{\max }(36 \mathrm{~b})$ of the Lyapunov function $V(X)$ defining the size of the domain $\mathbb{D}$ are given by

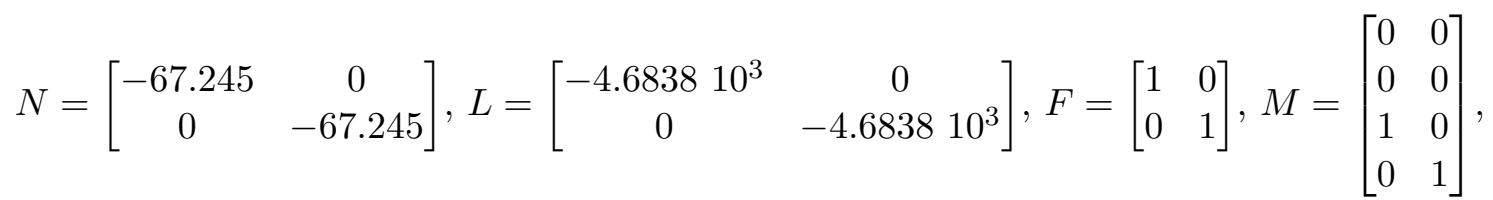

$$
\begin{aligned}
& \begin{array}{r}
E=\left[\begin{array}{cc}
1 & 0 \\
0 & 1 \\
69.682 & 0 \\
0 & 69.682
\end{array}\right], T=\left[\begin{array}{cccc}
-69.682 & 0 & 1 & 0 \\
0 & -69.682 & 0 & 1
\end{array}\right], K=\left[\begin{array}{cccc}
51.760 & 0 & 60.925 & 0 \\
0 & 51.760 & 0 & 60.925
\end{array}\right] \text { and } \\
V_{\max }=1.6145 .
\end{array}
\end{aligned}
$$


The initial conditions are set at

$$
\begin{gathered}
q_{1}(0)=0.1 \mathrm{rad}, q_{2}(0)=0.4 \mathrm{rad}, \dot{q}_{1}(0)=0 \mathrm{rad} \mathrm{s}^{-1}, \dot{q}_{2}(0)=0 \mathrm{rad} \mathrm{s}^{-1} \\
z_{1}(0)=0 \mathrm{rad} \mathrm{s}^{-1} \text { and } z_{2}(0)=0 \mathrm{rad} \mathrm{s}^{-1}
\end{gathered}
$$

Figures 2 to 7 in the appendix show a tracking simulation result. A perturbation is added to link 1 at time $2 \mathrm{~s}$ and to link 2 at time $4 \mathrm{~s}$ (see figure 4). As expected with the small value of $\gamma$, it can be noticed that the tracking error is small while the perturbations appear (see figures 5 and 6 ). When the perturbations disappear, the tracking errors go to zero with acceptable dynamics. Figure 7 shows the velocities of the robot and the "estimated" velocities for the two links. The "estimated" velocities converge on the robot velocities. The "estimated" velocities converge quickly on the robot velocities when the perturbations disappear.

\section{Conclusion}

In this paper, the trajectory tracking problem without velocity measurement has been discussed for an $\mathrm{n}$-joint rigid robot manipulator via a reduced-order output feedback controller. This controller is characterized by an external disturbance attenuation in a $L_{2}$-gain sense and provides an asymptotic stability of the closed-loop system. Moreover, the controller gains are determined by solving two unilaterally-coupled algebraic Riccati inequalities. The closed-loop stability analysis is addressed via the Lyapunov theory, and a domain contained into the attraction domain is given. A simulation example is presented to show the feasibility and the effectiveness of the proposed approach.

\section{References}

[AST 94] Astolfi A., L. Lanari (1994). Disturbance attenuation and set-point regulation of rigid robots via $H_{\infty}$ control. 33rd IEEE Conference on Decision Control, Lake Buena Vista, Florida, 2578-2583.

[BER 93a] Berghuis H., H. Nijmeijer (1993). Global regulation of robots using only position measurements. Systems \& Control Letters, 21, 289-293.

[BER 93b] Berghuis H., H. Nijmeijer (1993). A passivity approach to controller-observer design for robots. IEEE Transactions on Robotics and Automation, 9, 740-753.

[BOY 94] Boyd S., L. El Ghaoui, E. Feron, V. Balakrishnan (1994). Linear Matrix Inequalities in System and Control Theory. 15, Studies in Applied Mathematics, SIAM.

[CAN 92] Canudas de Wit C., N. Fixot, K.J. Åström (1992). Trajectory tracking in robot manipulators via nonlinear estimated state feedback. IEEE Transactions on Robotics and Automation, 8, 138-144.

[DOY 89] Doyle J.C., K. Glover, P.P. Khargonekar, B.A. Francis (1989). State-space solutions to standard $H_{2}$ and $H_{\infty}$ control problems. IEEE Transactions on Automatic Control, 34, 831-847.

[EGH 95] El Ghaoui L., R. Nikoukhah, F. Delebecque (1995). LMITOOL : a package for LMI optimization. 34th IEEE Conference on Decision and Control, New Orleans, 3096-3101.

[GAH 95] Gahinet P., A. Nemirovski, A.J. Laub, M. Chilali (1995). The LMI control toolbox. 3rd European Control Conference, Rome, 3206-3211.

[HSU 94] Hsu C.S., X. Yu, H.H. Yeh, S.S. Banda (1994). $H_{\infty}$ compensator design with minimal order observers. IEEE Transactions on Automatic Control, 39, 1679-1681.

[ISI 92] Isidori A., A. Astolfi (1992). Disturbance attenuation and $H_{\infty}$-control via measurement feedback in nonlinear systems. IEEE Transactions on Automatic Control, 37, 1283-1293. 
[KHE 96] Khelfi M.F., M. Zasadzinski, M. Darouach, H. Rafaralahy, E. Richard (1996). Reduced-order observer-based point-to-point and trajectory controllers for robot manipulators. Control Engineering Practice, 4, 991-1000.

[KNO 93] Knobloch H.W., A. Isidori, D. Flockerzi (1993). Topics in Control Theory. 22, DMV Seminar Band, Birkhuser.

[KRE 94] Krener A.J. (1994). Necessary and sufficient conditions for nonlinear worst case $\left(H_{\infty}\right)$ control and estimation. Journal of Mathematical Systems, Estimation, and Control, 4, 1-25.

[LEW 93] Lewis F.L., C.T. Abdallah, D.M. Dawson (1993). Control of Robot Manipulators, Macmillan.

[NIC 93] Nicosia S., P. Tomei (1990). Robot control by using only joint position measurements. IEEE Transactions on Automatic Control, 35, 1058-1061.

[SAM 90] Sampei M., T. Mita, M. Nakamichi (1990). An algebraic approach to $H_{\infty}$ output feedback control problems. Systems \& Control Letters, 14, 13-24.

[SPO 89] Spong M.W., M. Vidyasagar (1989), Robot Dynamics and Control, John Wiley \& Sons.

[VDS 92] van der Schaft A.J. (1992). $L_{2}$-Gain analysis of nonlinear systems and nonlinear state feedback $H_{\infty}$ control. IEEE Transactions on Automatic Control, 37, 770-784.

[VDS 96] van der Schaft A.J. (1996). L L $^{-G a i n}$ and Passivity Techniques in Nonlinear Control. 218, Lecture Notes in Control and Information Sciences, Springer-Verlag.

[ZAS 96] Zasadzinski M., M.F. Khelfi, M. Darouach, E. Richard (1996). Disturbance attenuation via a reduced-order output feedback controller for rigid robot manipulators. 13th World IFAC Congress, San Francisco, A, 403-408.

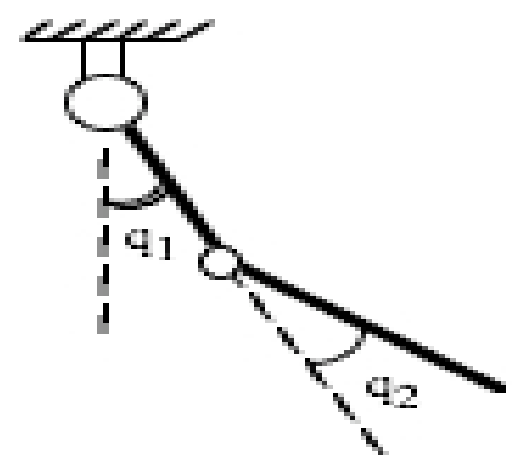

Figure 1: 2-D.O.F robot system. 


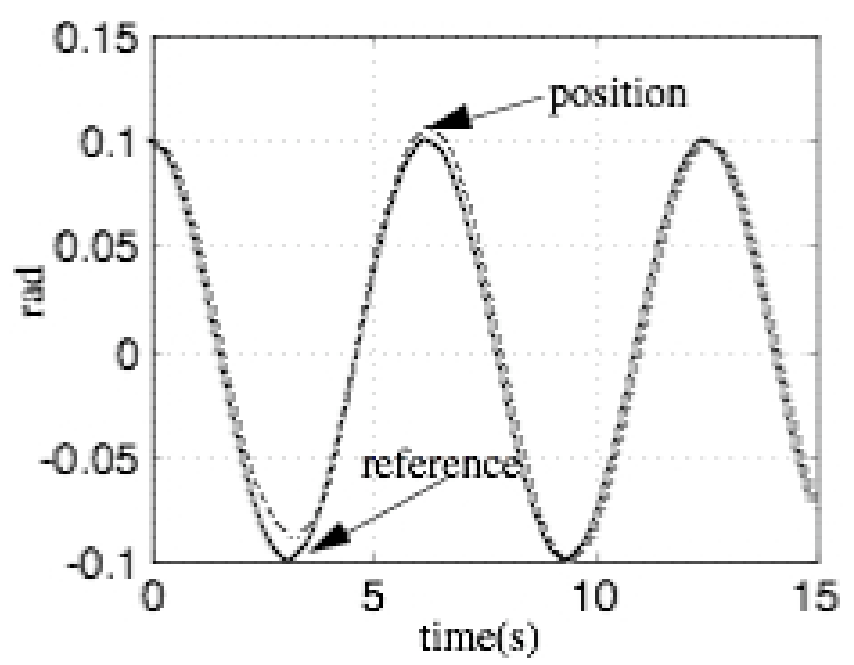

Figure 2: Reference and position, link 1.

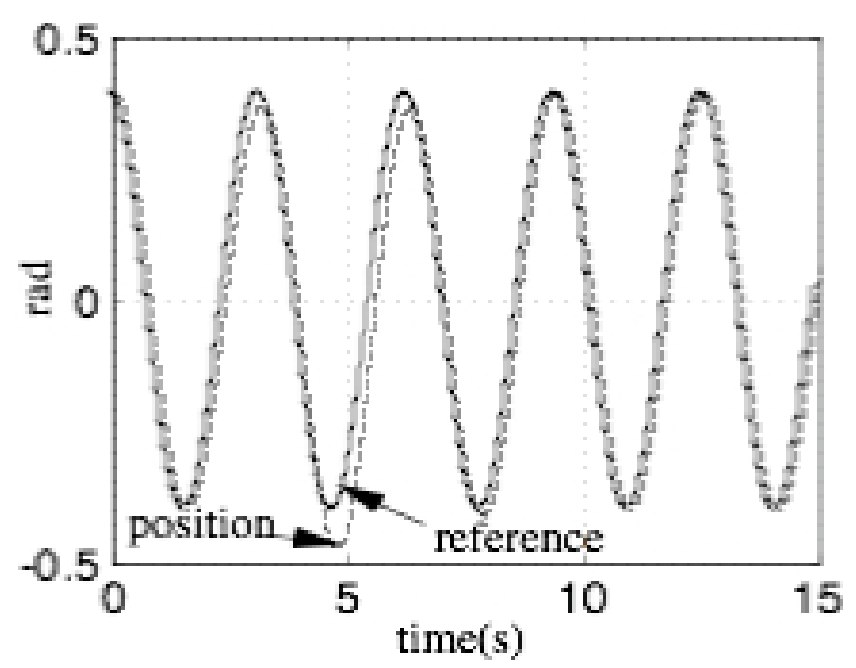

Figure 3: Reference and position, link 2.

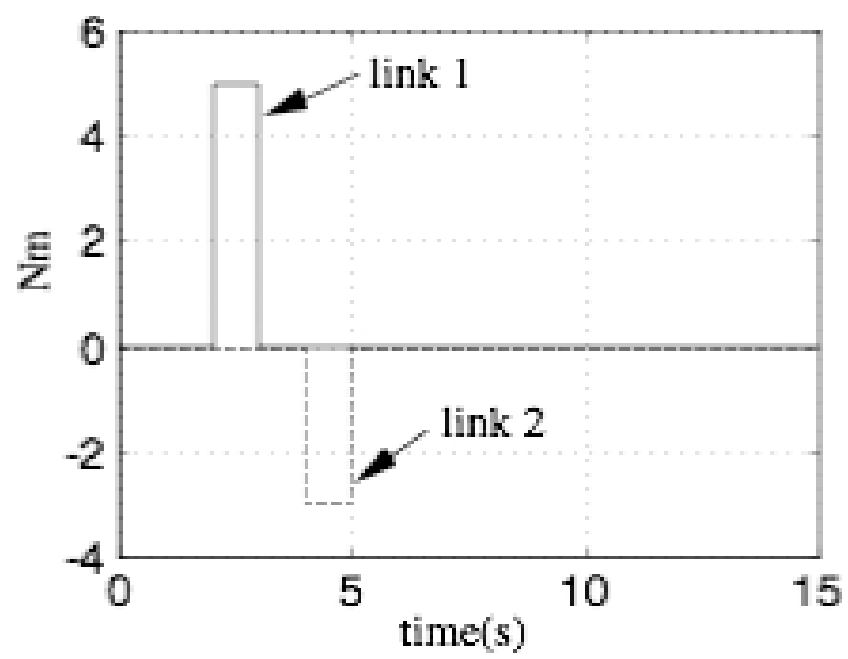

Figure 4: Perturbations, links 1 and 2. 


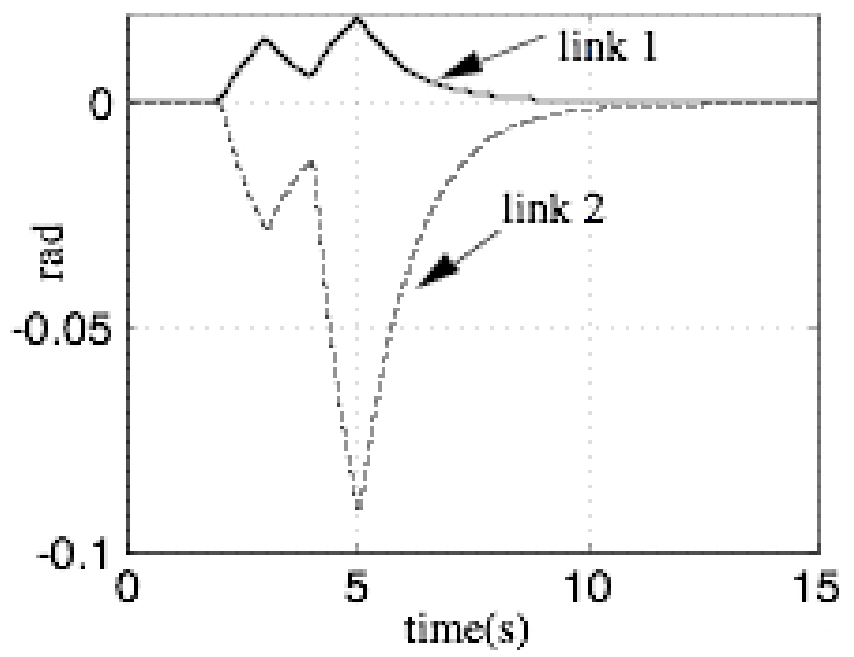

Figure 5: Position errors, links 1 and 2.

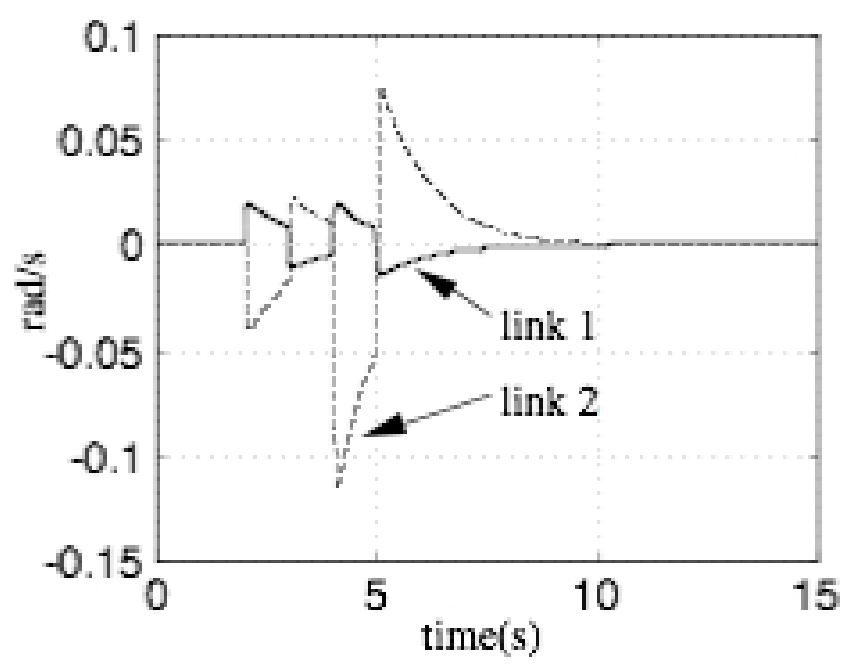

Figure 6: Velocity errors, links 1 and 2.

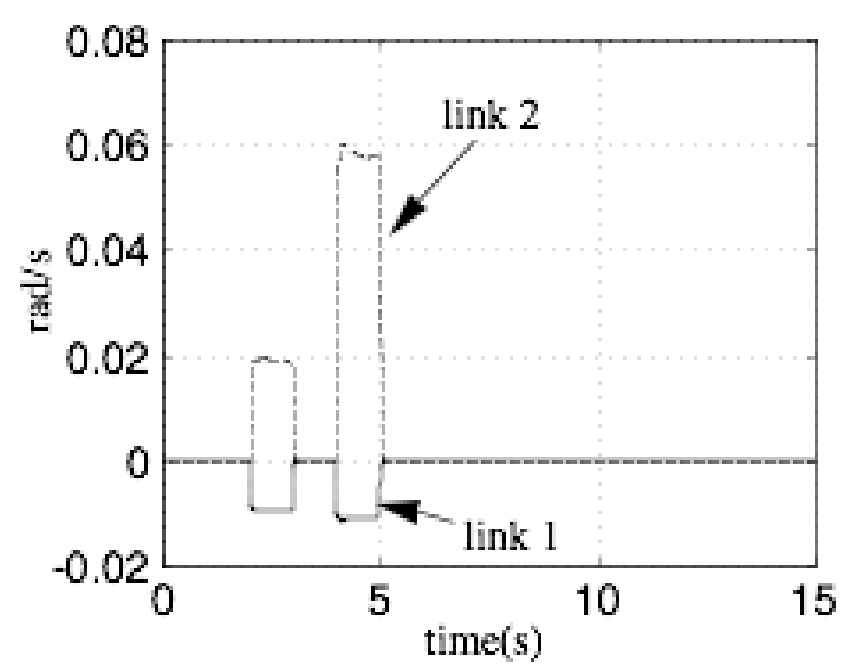

Figure 7: Velocity observation errors, links 1 and 2. 